\title{
Personality and the retention of neophobic predator avoidance in wild caught Trinidadian guppies
}

\author{
Grant E. Brown $^{\text {a,* }}$, Pierre J.C. Chuard ${ }^{\text {a }}$, Ebony E.M. Demers ${ }^{\text {a }}$, \\ Indar W. Ramnarine ${ }^{b}$, Douglas P. Chivers ${ }^{c}$ and Maud C.O. Ferrari ${ }^{\mathrm{d}}$ \\ ${ }^{a}$ Department of Biology, Concordia University, 7141 Sherbrooke Street West, Montreal, \\ QC, Canada H4B 1R6 \\ ${ }^{\mathrm{b}}$ Department of Life Sciences, University of the West Indies, St. Augustine, Republic of \\ Trinidad and Tobago \\ ${ }^{c}$ Department of Biology, University of Saskatchewan, 112 Science Place, Saskatoon, SK, \\ Canada S7N 1E2 \\ ${ }^{\mathrm{d}}$ Department of Biomedical Sciences, WCVM, University of Saskatchewan, 52 Campus \\ Drive, Saskatoon, SK, Canada S7N 5B4 \\ *Corresponding author's e-mail address: grant.brown@concordia.ca
}

Received 19 September 2016; initial decision 18 October 2016; revised 1 March 2017; accepted 3 March 2017; published online 4 April 2018

\begin{abstract}
Neophobic predator avoidance allows prey to reduce the risk of predation but is costly in terms of reduced foraging or courtship opportunities if the novel cues do not represent an actual threat. Consequently, neophobic responses to novel cues should wane with repeated exposures in the absence of an actual threat. We tested the prediction that individual personality traits shape the retention of neophobic predator avoidance in wild-caught guppies. Using extinction trials, we demonstrate that personality (measured as latency to escape or approach a novel object) did not influence the initial response of wild-caught Trinidadian guppies to a novel odour; bolder and shyer guppies both exhibited similarly strong avoidance responses. However, after several exposures, shyer guppies maintain an avoidance response, and bolder guppies no longer respond. Our results highlight the complex nature of the antipredator algorithm of prey, whereby past experience, acute risk, and individual tactics shape neophobic predator avoidance patterns.
\end{abstract}

\section{Keywords}

threat-sensitive trade-offs, behavioural plasticity, predator-prey interactions, induced neophobia, Trinidadian guppies. 


\section{Introduction}

For most animals, predation is an unrelenting force that influences almost everything in their lives, from the shape of their bodies, to their age of maturity and senescence, the number of offspring they produce, when and where they can be active, and even how they respond to unknown animals in their environment (Appleton \& Palmer, 1988; Crowl \& Covich, 1990; Lima \& Dill, 1990; Brönmark \& Miner, 1992; Brown et al., 2013). The ability to exhibit such plasticity reflects the need to be flexible in the face of predators that are spatially and temporally variable. Neophobia, the fear of novel stimuli, provides an excellent example of such plasticity. Elevated predation risk over as little as a few days dramatically changes the expression of neophobia. Guppies (Poecilia reticulata), cichlids (Amatitlania nigrofasciata, Pelvicachromis taeniatus), damselfish (Pomacentrus chrysurus) and tadpoles (Lithobates sylvaticus) all show antipredator responses to novel cues when raised in high-risk environments, but fail to do so when raised in low-risk environments (Brown et al., 2013, 2015a; Chivers et al., 2014; Meuthen et al. 2016). The differential expression of neophobia likely reflects differential costs and benefits associated with uncertainty. The probability that an unknown animal in a high-risk environment is actually a predator is higher than the probability that an unknown animal in a low-risk environment is actually a predator.

Neophobia can be seen as a mechanism to reduce the costs associated with inappropriate behavioural decisions until prey can acquire relevant information regarding local risk. However, responding to novel cues is not without costs. Continued avoidance of novel cues in the absence of an actual threat would result in, for example, lost foraging or mating opportunities. Brown et al. (2015b) recently explored the interactions between background risk and acute experience (presence vs. absence of reinforcements) on the retention of neophobic predator avoidance. Juvenile convict cichlids repeatedly exposed to the same cue at 3 day intervals for 15 days lose their neophobic response faster than conspecifics that are exposed only once over the same period of time. In the absence of a negative stimulus (i.e., presence of actual predator or other threatening cues), prey appear to learn that the stimulus is not threatening following repeated exposures (Brown et al., 2015b) via a mechanism akin to latent inhibition (Allen et al. 2002; Rankin et al. 2009).

Prey animals show a remarkable degree of intraspecific variability in the tactics used to balance the trade-offs between predator avoidance (e.g., vig- 
ilance) and, for example, foraging patterns (Sih et al., 2004; Réale et al., 2007). Often referred to as 'personalities', a wealth of research demonstrates that prey may be consistently risk averse ('shyer') vs. risk prone ('bolder') in their behavioural decisions (Sih et al., 2004; Brown et al., 2005; Réale et al., 2007; Jones \& Godin, 2010). For example, the response of Trinidadian guppies to known high-risk cues (conspecific chemical alarm cues) is dependent upon individual personality (measured as latency to emerge from shelter); shyer guppies exhibit strong responses while bolder guppies appear indifferent to the same cue (Brown et al., 2014). However, Brown et al. (2014) found no impact of 'personality' on the neophobic response to a novel chemosensory cue; regardless of individual risk taking tactic, all guppies exposed to a novel cue exhibited a strong avoidance response.

However, it remains unknown if the personality of prey would influence the retention of neophobic predator avoidance. Recent reports suggest a link between individual personality and learning abilities among prey (Carere \& Locurto, 2011; Sih \& Del Giudice, 2012). For example, bolder guppies were quicker to learn a novel foraging task and relied more heavily on social information to do so than were shyer conspecific (Trompf \& Brown, 2014). Bolder individuals are likely more active (Harris et al., 2010) and may have greater opportunities to learn ecologically relevant information (Coleman et al., 2005; Trompf \& Brown, 2014) compared to shyer conspecifics. Thus, we might expect neophobic predator avoidance to wane faster among bolder individuals. To test this hypothesis, we collected guppies from two high predation risk (i.e., neophobic) populations and using a repeated measures design, exposed focal shoals to either a novel chemosensory cue (vs. a water control) to test the retention of neophobic predator avoidance. We also recorded measures of individual risk-taking tactics for shoals at each observation. We predict that guppies should exhibit strong neophobic predator avoidance on their initial exposure to a novel chemical cue regardless of personality tactic. We also predict that after repeated exposure to the same cue, bolder guppies should lose their neophobic avoidance of the 'novel' cue faster than shyer guppies.

\section{Methods}

\subsection{Fish collection and stimulus}

We collected female guppies from two populations, the Lopinot and Lower Aripo Rivers, in the Northern Range Mountains, Trinidad, during April 2014. 
Both rivers contain a variety of piscivorous fishes, including pike cichlids (Crenicichla sp.), blue acara (Andinocara pulcher), brown coscorub ( $\mathrm{Ci}$ chlasoma taenia), and wolfish (Hoplias malabaricus). As such, both are considered high predation populations. Guppies from each population were collected from several different pools using $1 \mathrm{~m}$ hand seine nets and transported to the laboratory at the University of the West Indies, Saint-Augustine, Trinidad and Tobago. Guppies were held, separated by population, in aerated and continuously filtered 100-1 holding tanks (approximately $25^{\circ} \mathrm{C}, 12: 12$ L:D cycle) for at least 3 days prior to testing. We fed guppies, twice daily, with flake food and freeze-dried Tubifex.

We generated a novel chemosensory cue using Badia ${ }^{\mathrm{TM}}$ (Badia, Doral, FL, USA) pure lemon extract. We diluted the extract in dechlorinated tap water ( $6 \mathrm{ml}$ lemon extract in $300 \mathrm{ml}$ water; 'lemon odour'). Previous studies have shown that Trinidadian guppies from high predation populations (but not low predation populations) exhibit neophobic avoidance of this cue in laboratory and field trials (Brown et al., 2013, 2015b). As such, we did not include guppies from low predation risk populations in this experiment. Fresh lemon odour solutions were prepared for each round of trials (see below).

\subsection{Experimental protocol}

Test tanks consisted of a series of 20-1 glass aquaria, filled with 181 of dechlorinated tap water (approximately $26^{\circ} \mathrm{C}$ ). Each tank was aerated, but not filtered and contained a single airstone affixed along the back wall of the tank. We attached an additional length $(1.5 \mathrm{~m})$ of airline tubing, terminating immediately above the airstone, to facilitate the introduction of test stimuli without disturbing the focal fish. Shoals of 3 female guppies, haphazardly sampled from the holding tanks, were transferred to testing tanks $24 \mathrm{~h}$ prior to the initial observation. Using a repeated-measures design, we observed each shoal four times (at approximately 2.5 -h intervals) between 0800 and 1700. Each observation (trial) involved a measure of personality trait, immediately followed by a measure of a predator avoidance response to either a novel chemosensory cue (lemon odour) or a water control. Though guppies were not tagged, we were able to reliably assess the same focal guppy for the assessment of personality traits within each shoal based on size differences within shoals.

We quantified the latency to emerge from an acclimation zone (observations 1 and 3 ) or the latency to approach a novel object (observations 2 
and 4) as measures of personality traits. For latency to emerge, we positioned an opaque barrier approximately $10 \mathrm{~cm}$ from the back wall of the test tank. The focal shoal was confined to the acclimation zone for a period of 10 min prior to commencing an observation. We raised the barrier $2.5 \mathrm{~cm}$ and recorded the latency until the first (focal) guppy fully emerged. Once the focal guppy emerged, we gently removed the barrier and began the predator avoidance observation (see below). Shoals were given a maximum of 5 min to emerge, at which point the barrier was removed and the latency to emerge was recorded as $300 \mathrm{~s}$. For latency to approach, we positioned a novel object (green and white Lego ${ }^{\mathrm{TM}}$ blocks) affixed to a length of clear fishing line in the front third of the tank. We recorded the latency (maximum $300 \mathrm{~s}$ ) until the focal guppy exhibited a directed approach to within 3 body lengths of the stimulus. As above, once the focal guppy approached, we carefully removed the novel object with the attached line. Both measures have been previously shown to be reliable assays for measuring boldness vs. shyness for juvenile convict cichlids (Jones \& Godin, 2010). Using two different measures reduces the likelihood that focal shoals would acclimate to the test conditions.

Predator avoidance observations consisted of 5-min pre- and 5-min poststimulus injection periods. Following the emergence/approach measurements, we allowed a brief (1-2 min) period to ensure the guppies were not disturbed by the removal of the barrier or novel object. We then began the 5-min pre-stimulus observation periods.

Immediately following the pre-stimulus period, we injected $10 \mathrm{ml}$ of either lemon odour (novel cue) or dechlorinated tap water (control). Shoals were tested to only one of the two stimuli. During both pre- and post-stimulus period, we recorded an index of shoaling and area use every $15 \mathrm{~s}$. Shoaling index scores ranged from 1 (no fish within 1 body length of another) to 3 (all fish within 1 body length of another). Area use was recorded as the horizontal position of each fish within the tank. Scores ranged from 3 (all fish in the bottom third of the tank) to 9 (all fish in the top third of the tank). An increase in shoaling index and a reduction in horizontal area use are reliable indicators of antipredator behaviour in Trinidadian guppies (Brown et al. 2009; 2013). Following each round of testing, we conducted partial (75\%) water changes on each tank. We conducted a total of 30 observations per population for each of the treatment combinations $(N=120 \times 4$ repeated observations). 


\subsection{Statistical analysis}

Initially, we tested repeatability of our 'personality' measures using intraclass correlations. For antipredator behaviour, we calculated difference scores (post-stimulus-pre-stimulus) and used these as dependent variables in a repeated measures GLM. As a measure of risk taking tactic (i.e., 'personality'), we calculated the mean latency across the four observations for each shoal and included the mean latency score (log transformed) as a covariate as a proxy for personality. Stimulus (lemon odour vs. water) and population (Lower Aripo vs. Lopinot) as independent factors. All analyses were conducted using SPSS V23.0.

\subsection{Ethical note}

All work reported was conducted in accordance with Concordia University Animal Research Ethics protocol \#30000255. All work was conducted under laboratory conditions. Stock holding tanks contained environmental enrichment. All tanks (stock and testing tanks) were monitored daily, in accordance with Canadian Council on Animal Care, for water quality and general animal health. Guppies were collected under permit issued by the Ministry of Agriculture, Land and Fisheries, Fisheries Division, Republic of Trinidad and Tobago.

\section{Results}

We found significant intraclass correlations for guppies repeatedly exposed to distilled water (ICC: $0.69, \mathrm{CI}=0.55-0.81, p<0.001$ ) and lemon odour (ICC: $0.57, \mathrm{CI}=0.36-0.73, p<0.001$ ), suggesting that our measures were indeed highly repeatable.

Our RM-GLM found significant main effects for latency $\left(F_{1,114}=10.61\right.$, $\left.p<0.001, \eta_{p}^{2}=0.16\right)$ and stimulus $\left(F_{2,114}=51.44, p<0.001, \eta_{p}^{2}=0.47\right)$. There was no significant main effect of population $\left(F_{2,114}=1.04, p=0.36\right)$, nor a stimulus $\times$ population interaction $\left(F_{2,114}=1.66, p=0.19\right)$. Of particular interest, we found a significant observation number $\times$ stimulus $\times$ latency interaction $\left(F_{3,339}=4.72, p=0.003, \eta_{p}^{2}=0.04\right)$. While bolder and shyer guppies exhibit similar responses to the novel odour during the first exposure (Figures $1 \mathrm{~A}$ and $2 \mathrm{~A}$ ), by the final exposure, only shyer guppies show a response (Figures $1 \mathrm{~B}$ and $2 \mathrm{~B}$ ). 

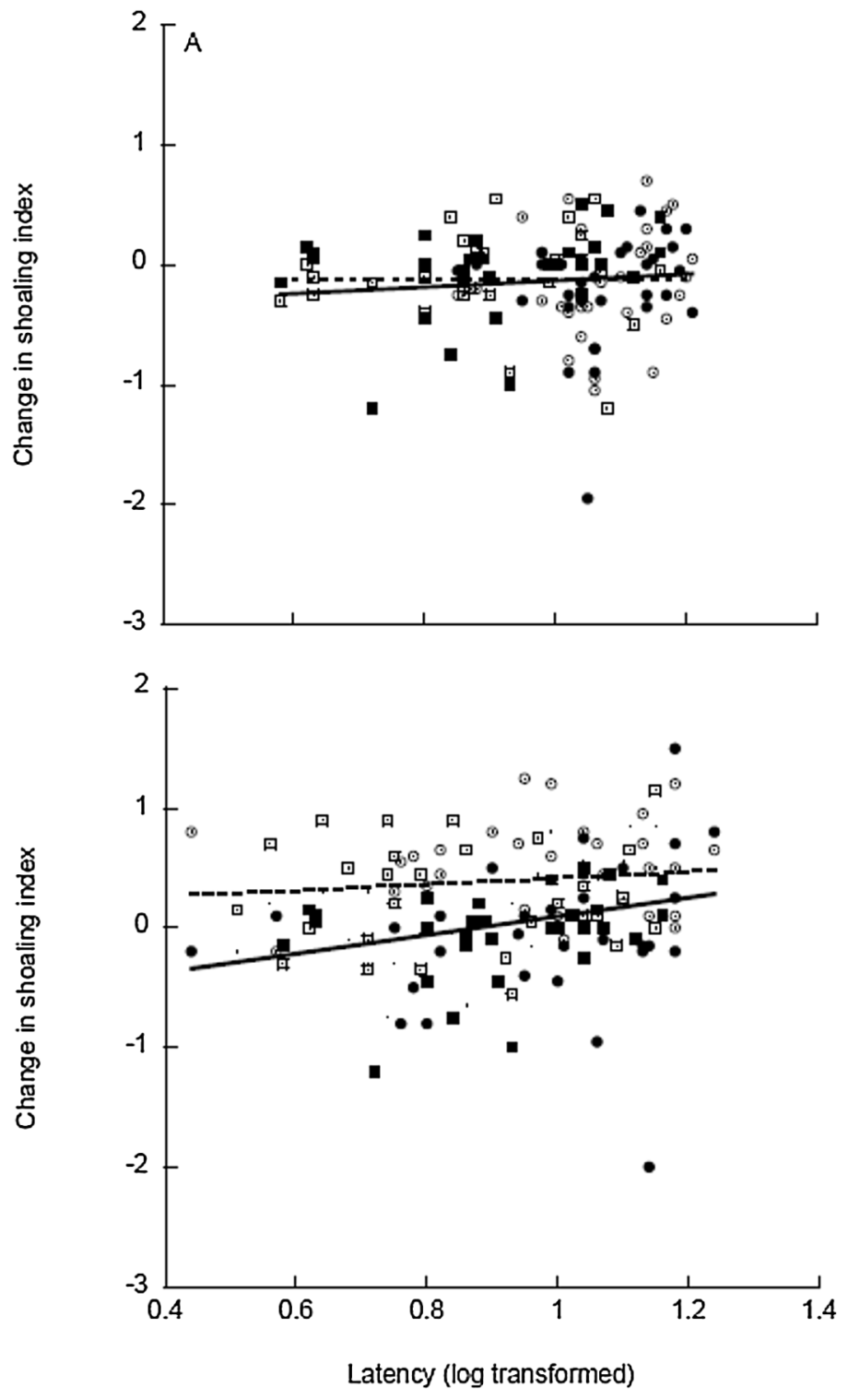

Figure 1. Change in shoaling index for Lower Aripo (circles) and Lopinot River (boxes) guppies during the first exposure (open symbols; dashed line) vs. fourth exposure (filled symbols, solid line), plotted in relation to mean latency scores (log transformed). $N=30$ per stimulus for each population tested. (A) Response of guppies to distilled water control; (B) response of guppies to lemon odour (novel cue). Lines are best fit lines for both populations combined. 

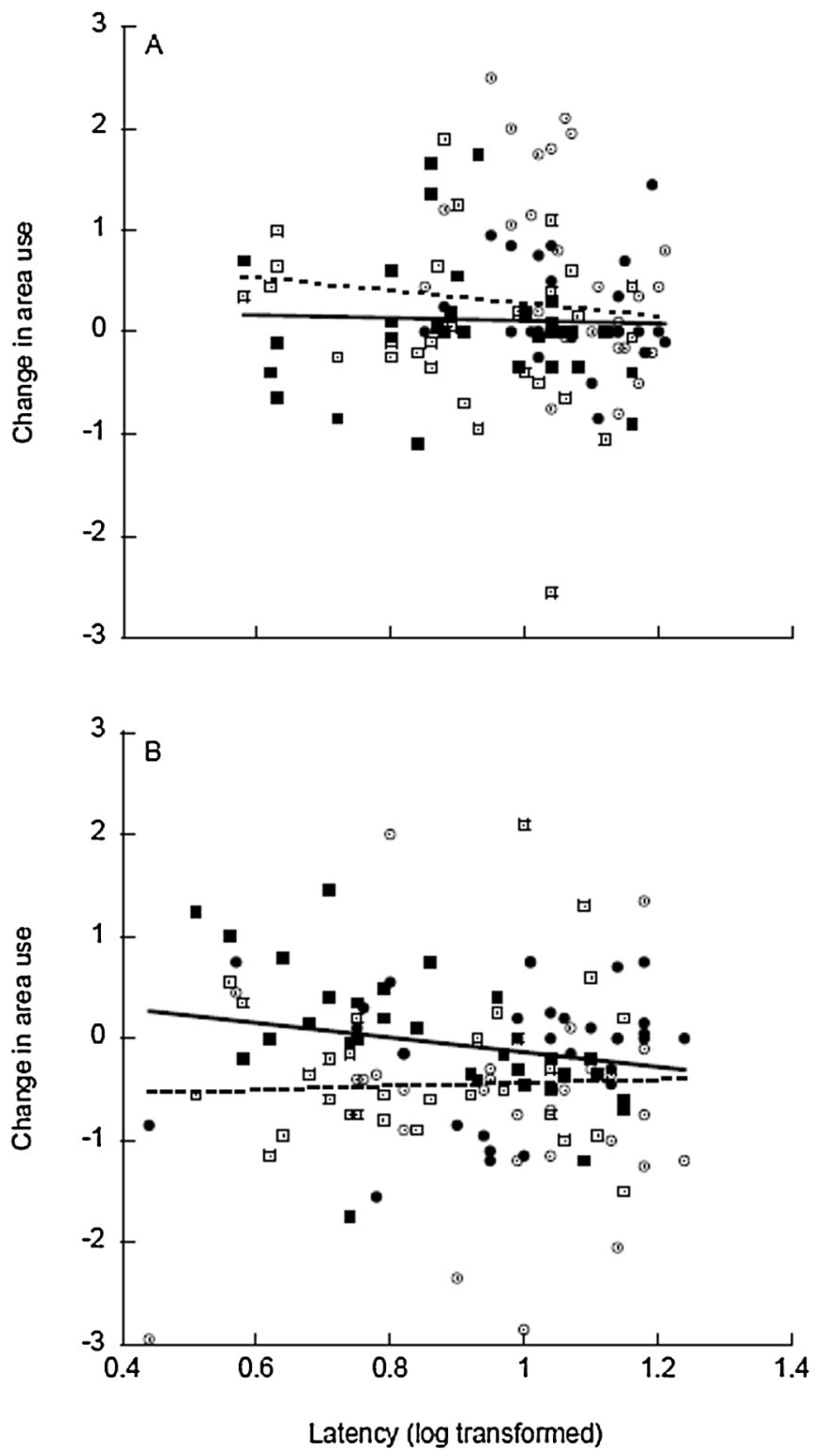

Figure 2. Change in area use for Lower Aripo (circles) and Lopinot River (boxes) guppies during the first exposure (open symbols, dashed line) vs. fourth exposure (filled symbols, solid line), plotted in relation to mean latency scores (log transformed). $N=30$ per stimulus for each population tested. (A) Response of guppies to distilled water control; (B) response of guppies to lemon odour (novel cue). Lines are best fit lines for both populations combined. 


\section{Discussion}

The results of our study suggest that personality traits affect the retention of neophobic responses. Initially, we found consistent risk taking tactics across the four repeated observations, consistent with previously documented repeatability of risk-taking tactics in Trinidadian guppies (Harris et al., 2010; Irving \& Brown, 2013; Trompf \& Brown, 2014). We also found no overall difference between the Lower Aripo and Lopinot River populations. This is not surprising as both can be considered high predation risk populations and have similar predator guilds (personal observations). Similar to the findings of Brown et al., 2014, when guppies were first exposed to lemon odour as a novel chemosensory cue, we found no effect of individual risk-taking tactics on their antipredator response intensity. Regardless of whether fish were bold or shy, they exhibited a strong avoidance response (compared to the water control) to lemon odour.

However, by the fourth presentation, bolder guppies no longer responded to lemon odour (i.e., were not different from the water control) while shyer guppies continued to show strong avoidance. It is important to note that the repeated exposures and measures of personality tactics were conducted over the course of a single day. As such, our current results do not allow us to differentiate between the number of repeated exposures vs. the rate of exposure. It is possible that if the latency between exposures was longer, the response of shyer guppies may have waned after a few exposures. Regardless, our results provide the first demonstration of personality influencing the retention of a neophobic response.

Previous studies have shown that in the absence of an actual predation threat, the neophobic response of prey animals to novel cues will quickly wane (Brown et al., 2015a, b). Similar to the retention of learned information (Ferrari et al., 2010a, b; Gonzalo et al., 2010; Brown et al., 2012), the retention of neophobic responses are likely subject to cost-benefit tradeoffs. Responding to cues that are not representative of an acute risk would represent a short-term cost (e.g., lost opportunity to forage, court, defend territories). Given the dramatic cost of not responding to a potential predator, prey likely benefit from exhibiting an initial neophobic response; it is better to suffer a lost foraging opportunity than to fail to respond to a predator. However, repeatedly responding in the absence of an actual threat would result in an accumulation of lost opportunity costs. 
There are several, non-mutually exclusive mechanisms that may account for the observed differences in retention of neophobic responses among shyer vs. bolder guppies. Our current data are consistent with the hypothesis that bolder vs. shyer individuals balance threat-sensitive trade-offs differently (Jones \& Godin, 2010; Pellegrini et al., 2010; Mathot et al., 2012). Compared to shyer individuals, bolder guppies may become less risk averse in favour of continued foraging. For example, Chapman et al. (2010) demonstrated that under conditions of unpredictable foraging opportunities, guppies engage in riskier foraging tactics and are more likely to explore novel foraging patches (i.e., are 'bolder') than conspecifics held under more predictable conditions. Likewise, strong interactions between bolder personality types and feeding motivations have been demonstrated (Brown et al., 2005; Biro \& Booth, 2009; David et al., 2012). Thus, an increase in the relative value of continued foraging and/or a decrease in the perceived risk among bolder vs. shyer individuals would shift the balance of predator-avoidance/foraging trade-offs. Secondly, individual risk-taking tactics may alter the integration of multiple sources of risk assessment information. Prey capable of integrating multiple cues (i.e., sensory complementarity; Elvidge et al., 2012) may benefit from more reliable risk assessments, particularly under conditions of uncertain predation risk (Munoz \& Blumstein, 2012). Given that bolder prey are more likely to visually inspect potential threats (Harris et al., 2010; Jones \& Godin, 2010; Pellegrini et al., 2010), personality may shape the relative value of complimentary or confirming risk assessment information (Brown et al., 2014). Bolder guppies may use the lack of an acute visual predator cue to devalue the perceived risk associated with the novel odour cue over repeated exposures. Guppies repeatedly exposed to the lemon odour stimulus in the absence of an actual predator would effectively be learning that the cues are not risky. Potential differences in the use of complimentary information among different personality types might explain the more rapid extinction among bolder guppies. Finally, recent reports suggest that individual personality may shape the cognitive abilities of prey (Carere \& Locurto, 2011; Sih \& Del Giudice, 2012). Bolder guppies, for example, learn novel foraging tasks faster and more accurately than do shyer conspecifics (Dugatkin \& Alfieri, 2003; Trompf \& Brown, 2014), likely resulting from increased exploration among bolder prey leading to enhanced encounters with novel patches (Trompf \& Brown, 2014). Similarly, bolder adult Japanese macaques (Macaca fuscata) are faster to learn a novel task reinforced with 
food than are shyer macaques (Coleman et al., 2005). Bolder male great tits (Parus major) are more efficient at acquiring a novel foraging task compared to shyer males (Titulaer et al., 2012). Given that bolder guppies are likely more active (Harris et al., 2010), they may have greater reliance on current or recent information (Trompf \& Brown, 2014). Likewise, increased activity would afford greater opportunity to learn that lemon odour was not risky (see above). Several researchers have demonstrated that conditions of high predation risk, perhaps counter-intuitively, induces bolder phenotypes among prey species (Brown et al., 2005; Bell \& Sih, 2007; Dingemanse et al., 2009). Given that bolder phenotypes may suffer higher predation risks (Biro et al., 2004) and foraging demands (Brown \& Braithwaite, 2004; Millot et al., 2009), the potential costs associated with making an inappropriate decision may be higher for bolder than shyer prey (Mathot et al., 2012; Brown et al., 2014). Regardless of the underlying mechanism, our current results suggest that bolder individuals may benefit from showing an initial neophobic response (short-term avoidance of risk) and fine-tune their behavioural decisions with repeated exposures to a 'novel' cue. As such, our results highlight the role past experience with predation risk, recent experience (acute risk) and individual risk-taking tactics play in shaping the neophobic predator avoidance patterns of prey animals.

\section{Acknowledgements}

We wish to thank Drs. James Grant, Jean-Guy Godin and Robert Weladji for providing useful insights and statistical advice. Harrison Désormeaux kindly provided us with the Lego stimulus. Financial support was provided by the Natural Sciences and Engineering Research Council of Canada and Concordia University to GEB. EEMD was supported by a FQRNT graduate fellowship. PJCC was supported by Concordia University Graduate Fellowship.

\section{References}

Allen, M.T., Chelius, L., Masand, V., Gluck, M.A., Myers, C.E. \& Schnirman, G. (2007). A comparison of latent inhibition and learned irrelevance pre-exposure effects in rabbit and human eyeblink conditioning. — Integr. Phys Behav. Sci. 37: 188-214.

Appleton, R.D. \& Palmer, R. (1988). Water-borne stimuli released by predatory crabs and damaged prey induce more predator-resistant shells in a marine gastropod. - Proc. Natl Acad. Sci. USA 85: 4387-4391. 
Bell, A.M. \& Sih, A. (2007). Exposure to predation generates personality in threespined sticklebacks (Gasterosteus aculeatus). - Ecol. Lett. 10: 828-834.

Biro, P.A. \& Booth, D.J. (2009). Extreme boldness precedes starvation mortality in six-lined trumpeter (Pelates sexlineatus). - Hydrobiology 635: 395-398.

Biro, P.A., Abrahams, M.V., Post, J.R. \& Parkinson, E.A. (2004). Predators select against high growth rates and risk-taking behaviour in domestic trout populations. - Proc. Roy. Soc. Lond. B: Biol. Sci. 271: 2233-2337.

Brönmark, C. \& Miner, J.G. (1992). Predator-induced phenotypical change in body morphology in crucian carp. - Science 258: 1348-1350.

Brown, C. \& Braithwaite, V.A. (2004). Size matters: a test of boldness in eight populations of the poeciliid Brachyraphis episcopi. - Anim. Behav. 68: 1325-1329.

Brown, C., Jones, F. \& Braithwaite, V.A. (2005). In situ examination of boldness-shyness traits in the tropical poeciliid, Brachyraphis episcopi. - Anim. Behav. 70: 1003-1009.

Brown, G.E., Macnaughton, C.J., Elvidge, C.K., Ramnarine, I. \& Godin, J.-G.J. (2009). Provenance and threat-sensitive predator avoidance patterns in wild-caught Trinidadian guppies. — Behav. Ecol. Sociobiol. 63: 699-706.

Brown, G.E., Ferrari, M.C.O., Malka, P.H., Fregeau, L., Kayello, L. \& Chivers, D.P. (2012). Retention of acquired predator recognition among shy versus bold juvenile rainbow trout. — Behav. Ecol. Sociobiol. 67: 43-51.

Brown, G.E., Ferrari, M.C.O., Elvidge, C.K., Ramnarine, I. \& Chivers, D.P. (2013). Phenotypically plastic neophobia: a response to variable predation risk. — Proc. Roy. Soc. Lond. B: Biol. Sci. 280: 20122712.

Brown, G.E., Elvidge, C.K., Ramnarine, I., Chivers, D.P. \& Ferrari, M.C.O. (2014). Personality and the response to predation risk: effects of information quantity and quality. Anim. Cogn. 17: 1063-1069.

Brown, G.E., Elvidge, C.K., Ramnarine, I., Ferrari, M.C.O. \& Chivers, D.P. (2015a). Background risk and recent experience influences retention of neophobic responses to predators. - Behav. Ecol. Sociobiol. 69: 737-745.

Brown, G.E., Demers, E.E., Joyce, B.J., Ferrari, M.C.O. \& Chivers, D.P. (2015b). Retention of neophobic predator recognition in juvenile convict cichlids: effects of background risk and recent experience. - Anim. Cogn. 18: 1331-1338.

Carere, C. \& Locurto, C. (2011). Interaction between animal personality and animal cognition. - Curr. Zool. 57: 491-498.

Chapman, B.B., Morrell, L.J. \& Krause, J. (2010). Unpredictability in food supply during early life influences boldness in fish. — Behav. Ecol. 21: 501-506.

Chivers, D.P., McCormick, M.I., Mitchell, M.D., Ramasamy, R.A. \& Ferrari, M.C.O. (2014). Background level of risk determines how prey categorize predators and non-predators. Proc. Roy. Soc. Lond. B: Biol. Sci. 281: 20140355.

Coleman, K., Tully, L.A. \& McMillan, J.L. (2005). Temperament correlates with training success in adult rhesus macaques. - Am. J. Primatol. 65: 63-71.

Crowl, T.A. \& Covich, A.P. (1990). Predator-induced life-history shifts in a freshwater snail. — Science 247: 949-951. 
David, M., Auclair, Y., Giraldeau, L.-A. \& Cézilly, F. (2012). Personality and body condition have additive effects on motivation to feed in zebra finches Taeniopygia guttata. — Ibis 154: $372-378$.

Dingemanse, N.J., Van der Plas, F., Wright, J., Réale, D., Schrama, M., Roff, D.A., Van der Zee, E. \& Barber, I. (2009). Individual experience and evolutionary history of predation affect expression of heritable variation in fish personality and morphology. Proc. Roy. Soc. Lond. B: Biol. Sci. 276: 1285-1293.

Dugatkin, L.A. \& Alfieri, M.S. (2003). Boldness, behavioral inhibition and learning. - Ethol. Ecol. Evol. 15: 43-49.

Elvidge, C.K., Macnaughton, C.J. \& Brown, G.E. (2012). Sensory complementation and antipredator behavioural compensation in acid-impacted juvenile Atlantic salmon. - Oecologia 172: 69-78.

Ferrari, M.C.O., Brown, G.E., Bortolotti, G.R. \& Chivers, D.P. (2010a). Linking predator risk and uncertainty to adaptive forgetting: a theoretical framework and empirical test using tadpoles. — Proc. R. Soc. Lond. B Biol. 277: 2205-2210.

Ferrari, M.C.O., Brown, G.E., Jackson, C.D., Malka, P.H. \& Chivers, D.P. (2010b). Differential retention of predator recognition by juvenile rainbow trout. - Behaviour 147: 1791-1802.

Gonzalo, A., López, P. \& Martín, J. (2010). Risk level of chemical cues determines retention of recognition of new predators in Iberian green frog tadpoles. - Behav. Ecol. Sociobiol. 64: 1117-1123.

Harris, S., Ramnarine, I.W., Smith, H.G. \& Pettersson, L.B. (2010). Picking personalities apart: estimating the influence of predation, sex and body size on boldness in the guppy Poecilia reticulata. - Oikos 119: 1711-1718.

Irving, E. \& Brown, C. (2013). Examining the link between personality and laterality in a feral guppy Poecilia reticulata population. - J. Fish Biol. 83: 311-325.

Jones, K.A. \& Godin, J.-G.J. (2010). Are fast explorers slow reactors? Linking personality type and anti-predator behaviour. — Proc. Roy. Soc. Lond. B: Biol. Sci. 277: 625-632.

Lima, S.L. \& Dill, L.M. (1990). Behavioral decisions made under the risk of predation: a review and prospectus. — Proc. R. Soc. Lond. B Biol. Can. J. Zool. 68: 619-640.

Mathot, K.J., Wright, J., Kempenaers, B. \& Dingemanse, N.J. (2012). Adaptive strategies for managing uncertainty may explain personality-related differences in behavioural plasticity. - Oikos 121: 1009-1020.

Meuthen, D., Baldauf, S.A., Bakker, T.C.M. \& Thünken, T. (2016). Predator-induced neophobia in juvenile cichlids. - Oecologia 181: 947-958.

Millot, S., Begout, M.-L. \& Chatain, B. (2009). Exploration behaviour and flight response toward a stimulus in three sea bass strains (Dicentrarchus labrax L.). - Appl. Anim. Behav. Sci. 119: 108-114.

Munoz, N.E. \& Blumstein, D.T. (2012). Multisensory perception in uncertain environments. - Behav. Ecol. 23: 457-462.

Pellegrini, A.F.A., Wisenden, B.D. \& Sorensen, P.W. (2010). Bold minnows consistently approach danger in the field and lab in response to either chemical or visual indicators of predation risk. — Behav. Ecol. Sociobiol. 64: 381-387. 
Rankin, C.H., Abrams, T., Barry, R.J., Bhatnagar, S., Clayton, D.F., et al. (2009). Habituation revisited: an updated and revised description of the behavioral characteristics of habituation. - Neurobiol. Learn. Mem. 92: 135-138.

Réale, D., Reader, S.M., Sol, D., McDougall, P.T. \& Dingemanse, N.J. (2007). Integrating animal temperament within ecology and evolution. — Biol. Rev. 82: 291-318.

Sih, A. \& Del Giudice, M. (2012). Linking behavioural syndromes and cognition: a behavioural ecology perspective. - Phil. Trans. R. Soc., B 367: 2762-2772.

Sih, A., Bell, A.M., Johnson, J.C. \& Ziemba, R.E. (2004). Behavioral syndromes: an integrative overview. - Q. Rev. Biol. 79: 241-277.

Titulaer, M., van Oers, K. \& Naguib, M. (2012). Personality affects learning performance in difficult tasks in a sex-dependent way. — Anim. Behav. 83: 723-730. 